\title{
Robust stability of probabilistic delays fuzzy stochastic $p$-Laplace dynamic equations
}

Xiongrui Wang ${ }^{1}$ and Ruofeng Rao ${ }^{1,2^{*}}$

\section{*Correspondence:}

ruofengrao@163.com

${ }^{1}$ Institution of Mathematics, Yibin

University, Yibin, Sichuan 644007,

China

${ }^{2}$ Department of Mathematics,

Chengdu Normal University,

Chengdu, Sichuan 611130, China

\begin{abstract}
In this paper, the stability of a class of time-delay Takagi-Sugeno (T-S) fuzzy Markovian jumping partial differential equations (PDEs) with $p$-Laplace and probabilistic time-varying delays is investigated, and the robust exponential stability criterion is obtained by way of some variational methods in Sobolev space $W^{1, p}(\Omega)$, the Lyapunov functional method and the linear matrix inequalities technique. Moreover, a numerical example shows the effectiveness of the proposed methods due to the large allowable variation range of time delay.
\end{abstract}

Keywords: probabilistic time-varying delays; Markovian jumping; Takagi-Sugeno fuzzy mathematical model

\section{Introduction and preparation}

Given a complete probability space $(\boldsymbol{\Omega}, \mathcal{F}, \mathbb{P})$ with a natural filtration $\left\{\mathcal{F}_{t}\right\}_{t \geq 0}$, where $\boldsymbol{\Omega}$ is a sample space, $\mathcal{F}$ is $\sigma$-algebra of a subset of the sample space, and $\mathbb{P}$ is the probability measure defined on $\mathcal{F}$. Let $S=\{1,2, \ldots, N\}$ and the random form process $\{r(t), t \in[0,+\infty)\}$ be a homogeneous, finite-state Markovian process with right continuous trajectories with generator $\Pi=\left(\pi_{i j}\right)_{N \times N}$ and transition probability from mode $i$ at time $t$ to mode $j$ at time $t+\Delta t, i, j \in S, \mathbb{P}(r(t+\delta)=j \mid r(t)=i)=\pi_{i j} \delta+o(\delta)$ if $j \neq i$, and $\mathbb{P}(r(t+\delta)=j \mid r(t)=i)=$ $1+\pi_{i j} \delta+o(\delta)$ if $j=i$, where $\pi_{i j} \geq 0$ is transition probability rate from $i$ to $j(j \neq i)$ and $\pi_{i i}=-\sum_{j=1, j \neq i}^{s} \pi_{i j}, \delta>0$ and $\lim _{\delta \rightarrow 0} o(\delta) / \delta=0$.

Let us consider the following delayed Markovian jumping PDEs:

$$
\begin{aligned}
d u(t, x)= & {\left[\nabla \cdot\left(\mathcal{D}(t, x, u) \circ \nabla_{p} u(t, x)\right)-B(u(t, x))+C(r(t), t) f(u(t, x))+D(r(t), t)\right.} \\
& \times g(u(t-\tau(r(t), t), x))] d t+\sigma(u(t, x), u(t-\tau(r(t), t), x)) d w(t), \\
& t \geq 0, x \in \Omega
\end{aligned}
$$

equipped with the initial condition $u(\theta, x)=\phi(\theta, x),(\theta, x) \in[-\tau, 0] \times \Omega$ and zero-boundary condition

$$
\mathfrak{B}\left[u_{i}(t, x)\right]=0, \quad(t, x) \in[-\tau,+\infty) \times \partial \Omega, i=1,2, \ldots, n,
$$

where $w(t)$ is a standard one-dimensional Brownian motion defined on the probability space. $p>1$ is a positive scalar, $\Omega \in R^{m}$ is a bounded domain with a smooth boundary

(02014 Wang and Rao; licensee Springer. This is an Open Access article distributed under the terms of the Creative Commons Attribution License (http://creativecommons.org/licenses/by/2.0), which permits unrestricted use, distribution, and reproduction in any medium, provided the original work is properly cited. 
$\partial \Omega$ of class $\mathcal{C}^{2}$ by $\Omega, u(t, x)=\left(u_{1}(t, x), u_{2}(t, x), \ldots, u_{n}(t, x)\right)^{T} \in R^{n}$. In what follows, $u(t, x)$ is always denoted by $u$ for convenience sake. $\mathcal{D}(t, x, u) \circ \nabla_{p} u(t, x)$ denotes the Hadamard product of matrix $\mathcal{D}(t, x, u)$ and $\nabla_{p} u$ (see [1] or [2]), and $\mathcal{D}(t, x, v)=\left(\mathcal{D}_{j k}(t, x, u)\right)_{n \times m}$ satisfies $\mathcal{D}_{j k}(t, x, u) \geq 0$ for all $j, k,(t, x, u)$. In mode $r(t)=i \in S=\{1,2, \ldots, N\}$, we denote $C_{i}(t)=C(r(t), t)$ and $D_{i}(t)=D(r(t), t)$. Denote by $\tau_{i}(t)$ the time delay $\tau(r(t), t)$ which satisfies $0 \leq \tau_{i}(t) \leq \tau$ for any mode $i \in S$. Functions $B(u)=\left(B_{1}\left(u_{1}\right), B_{2}\left(u_{2}\right), \ldots, B_{n}\left(u_{n}\right)\right)^{T} \in R^{n}$, $f(u)=\left(f_{1}\left(u_{1}\right), f_{2}\left(u_{2}\right), \ldots, f_{n}\left(u_{n}\right)\right)^{T} \in R^{n}, g(u)=\left(g_{1}\left(u_{1}\right), g_{2}\left(u_{2}\right), \ldots, g_{n}\left(u_{n}\right)\right)^{T} \in R^{n}$. The boundary condition (1.1a) is called Dirichlet boundary condition if $\mathfrak{B}\left[u_{i}(t, x)\right]=u_{i}(t, x)$ and Neumann boundary condition if $\mathfrak{B}\left[u_{i}(t, x)\right]=\frac{\partial u_{i}(t, x)}{\partial v}$. Here, $\frac{\partial u_{i}(t, x)}{\partial v}=\left(\frac{\partial u_{i}(t, x)}{\partial x_{1}}, \frac{\partial u_{i}(t, x)}{\partial x_{2}}, \ldots, \frac{\partial u_{i}(t, x)}{\partial x_{m}}\right)^{T}$ denotes the outward normal derivative on $\partial \Omega$.

For mode $i \in S$, PDEs (1.1) is simply denoted as

$$
\begin{aligned}
d u= & {\left[\nabla \cdot\left(\mathcal{D}(t, x, u) \circ \nabla_{p} u\right)-B(u)+C_{i}(t) f(u)+D_{i}(t) g\left(u\left(t-\tau_{i}(t), x\right)\right)\right] d t } \\
& +\sigma\left(u, u\left(t-\tau_{i}(t), x\right)\right) d w(t), \quad t \geq 0, x \in \Omega .
\end{aligned}
$$

The T-S fuzzy mathematical model with time delay is described as follows.

Fuzzy rule $j$ :

IF $\omega_{1}(t)$ is $\mu_{j 1}$ and $\ldots \omega_{s}(t)$ is $\mu_{j s}$ THEN

$$
\begin{aligned}
d u= & {\left[\nabla \cdot\left(\mathcal{D}(t, x, u) \circ \nabla_{p} u\right)-B(u)+C_{i j}(t) f(u)+D_{i j}(t) g\left(u\left(t-\tau_{i}(t), x\right)\right)\right] d t } \\
& +\sigma\left(u, u\left(t-\tau_{i}(t), x\right)\right) d w(t),
\end{aligned}
$$

where $\omega_{k}(t)(k=1,2, \ldots, s)$ is the premise variable, $\mu_{j k}(j=1,2, \ldots, r ; k=1,2, \ldots, s)$ is the fuzzy set that is characterized by membership function, $r$ is the number of the

IF-THEN rules, and $s$ is the number of the premise variables.

For any mode $r(t)=i \in S$, we assume that $C_{i j}$, $D_{i j}$ are real constant matrices of appropriate dimensions, and $\Delta C_{i j}, \Delta D_{i j}$ are real-valued matrix functions which stand for timevarying parameter uncertainties, satisfying

$$
C_{i j}(t)=C_{i j}+\Delta C_{i j}(t), \quad D_{i j}(t)=D_{i j}+\Delta D_{i j}(t) .
$$

By way of a standard fuzzy inference method, system (1.3) is inferred as follows:

$$
\begin{aligned}
d u= & \left\{\nabla \cdot\left(\mathcal{D}(t, x, u) \circ \nabla_{p} u\right)-B(u)\right. \\
& \left.+\sum_{j=1}^{r} h_{j}(\omega(t))\left[C_{i j}(t) f(u)+D_{i j}(t) g\left(u\left(t-\tau_{i}(t), x\right)\right)\right]\right\} d t \\
& +\sigma\left(u, u\left(t-\tau_{i}(t), x\right)\right) d w(t),
\end{aligned}
$$

where $\omega(t)=\left[\omega_{1}(t), \omega_{2}(t), \ldots, \omega_{s}(t)\right], h_{j}(\omega(t))=\frac{w_{j}(\omega(t))}{\sum_{k=1}^{k} w_{k}(\omega(t))}, w_{j}(\omega(t)): R^{s} \rightarrow[0,1](j=$ $1,2, \ldots, r)$ is the membership function of the system with respect to the fuzzy rule $j . h_{j}$ can be regarded as the normalized weight of each IF-THEN rule, satisfying $h_{j}(\omega(t)) \geq 0$ and $\sum_{j=1}^{r} h_{j}(\omega(t))=1$. 
Next, we consider the following information for probability distribution of time delays $\tau_{i}(t)$ for all $i \in S$ :

$$
\mathbb{P}\left(0 \leq \tau_{i}(t) \leq \tau_{1 i}\right)=c_{0}, \quad \mathbb{P}\left(\tau_{1 i}<\tau_{i}(t) \leq \tau_{2 i}\right)=1-c_{0}
$$

Here the nonnegative scalar $c_{0} \leq 1$. Define a random variable as follows:

$$
\mathscr{C}(t)= \begin{cases}1, & 0 \leq \tau_{i}(t) \leq \tau_{1 i} \\ 0, & \tau_{1 i}<\tau_{i}(t) \leq \tau_{2 i}\end{cases}
$$

So, in this paper, we consider the following delayed Takagi-Sugeno (T-S) fuzzy Markovian jumping $p$-Laplace partial differential equations (PDEs) with probabilistic timevarying delays:

$$
\begin{aligned}
d u= & \nabla \cdot\left(\mathcal{D}(t, x, u) \circ \nabla_{p} u\right) d t \\
& -A(u)\left\{B(u)-\sum_{j=1}^{r} h_{j}(\omega(t))\left[C_{i j}(t) f(u)+c_{0} D_{i j}(t) g\left(u\left(t-\tau_{1 i}(t), x\right)\right)\right.\right. \\
& +\left(1-c_{0}\right) D_{i j}(t) g\left(u\left(t-\tau_{2 i}(t), x\right)\right)+\left(\mathscr{C}(t)-c_{0}\right)\left(D_{i j}(t) g\left(u\left(t-\tau_{1 i}(t), x\right)\right)\right. \\
& \left.\left.\left.-D_{i j}(t) g\left(u\left(t-\tau_{2 i}(t), x\right)\right)\right)\right]\right\} d t \\
& +\sigma\left(u, u\left(t-\tau_{1 i}(t), x\right), u\left(t-\tau_{2 i}(t), x\right), i\right) d w(t),
\end{aligned}
$$

where $0 \leq \tau_{1 i}(t) \leq \tau_{1 i}, \tau_{1 i}<\tau_{2 i}(t) \leq \tau_{2 i}$, and $\mathscr{C}(t)$ is the Bernoulli distributed sequence, satisfying $\mathbb{P}(\mathscr{C}(t)=1)=\mathbb{P}\left(0 \leq \tau_{1 i}(t) \leq \tau_{1 i}\right)=\mathbb{E}(\mathscr{C}(t))=c_{0}$, and $\mathbb{P}(\mathscr{C}(t)=0)=\mathbb{P}\left(\tau_{1 i}<\tau_{2 i}(t) \leq\right.$ $\left.\tau_{2 i}\right)=1-\mathbb{E}(\mathscr{C}(t))=1-c_{0}$. Here, $\mathbb{E}(\mathscr{C}(t))$ denotes the mathematical expectation of $\mathscr{C}(t)$. Note that the global existence of the solution of system (1.6) was investigated in [3]. To study the stability of (1.6), we need to assume

(A1) Let $A(u)=\operatorname{diag}\left(a_{1}\left(u_{1}(t, x)\right), a_{2}\left(u_{2}(t, x)\right), \ldots, a_{n}\left(u_{n}(t, x)\right)\right), \underline{A}=\operatorname{diag}\left(\underline{a}_{1}, \underline{a}_{2}, \ldots, \underline{a}_{n}\right)$, and $\bar{A}=\operatorname{diag}\left(\bar{a}_{1}, \bar{a}_{2}, \ldots, \bar{a}_{n}\right)$ such that $0<\underline{a}_{j} \leq a_{j}(r) \leq \bar{a}_{j}, j=1,2, \ldots, n$;

(A2) Let $B(u)=\left(B_{1}\left(u_{1}\right), B_{2}\left(u_{2}\right), \ldots, B_{n}\left(u_{n}\right)\right)^{T} \in R^{n}$, there exists a positive definite diagonal matrix $\mathbb{B}=\operatorname{diag}\left(b_{1}, b_{2}, \ldots, b_{n}\right)$ such that $\frac{B_{j}(r)}{r} \geq b_{j}, \forall j=1,2, \ldots, n$, and $0 \neq r \in R$

(A3) There exist constant diagonal matrices $G_{k}=\operatorname{diag}\left(G_{1}^{(k)}, G_{2}^{(k)}, \ldots, G_{n}^{(k)}\right)$, $F_{k}=\operatorname{diag}\left(F_{1}^{(k)}, F_{2}^{(k)}, \ldots, F_{n}^{(k)}\right), k=1,2$ with $\left|F_{j}^{(1)}\right| \leq F_{j}^{(2)},\left|G_{j}^{(1)}\right| \leq G_{j}^{(2)}, j=1,2, \ldots, n$, such that $F_{j}^{(1)} \leq \frac{f_{j}(r)}{r} \leq F_{j}^{(2)}, G_{j}^{(1)} \leq \frac{g_{j}(r)}{r} \leq G_{j}^{(2)}, \forall j=1,2, \ldots, n$, and $r \in R$.

(A4) There exist positive define symmetric matrices $\Gamma_{1 i}, \Gamma_{2 i}, \Gamma_{3 i}$ such that

$\operatorname{Trace}\left[\sigma^{T}(t) \sigma(t)\right] \leq u^{T} \Gamma_{1 i} u+u^{T}\left(t-\tau_{1 i}, x\right) \Gamma_{2 i} u\left(t-\tau_{1 i}, x\right)+u^{T}\left(t-\tau_{2 i}, x\right) \Gamma_{3 i} u\left(t-\tau_{2 i}, x\right)$, $i \in S$.

(A5) $\dot{\tau}_{k i}(t)+\sum_{l \in S} \pi_{i l} \tau_{k l}(t) \leq a_{0}<1$ for any mode $i \in S$, and $k=1,2$.

In addition, one can assume that $u=0$ is a trivial solution of PDEs (1.6) provided that $B(0)=f(0)=g(0)=0$. For any mode $i \in S$, the parameter uncertainties considered here are norm-bounded and of the following forms:

$$
\left(\Delta C_{i j}(t) \quad \Delta D_{i j}(t)\right)=E_{i j} \mathfrak{F}(t)\left(H_{i j} \quad M_{i j}\right), \quad \forall i \in S
$$


Here $\mathfrak{F}(t)$ is an unknown matrix function satisfying $\left|\mathfrak{F}^{T}(t) \| \mathfrak{F}(t)\right| \leq I$, and $E_{i j}, G_{i j}, H_{i j}$ are known real constant matrices. Throughout this paper, for a matrix $C=\left(c_{i j}\right)_{n \times n}$, we denote the matrix $|C|=\left(\left|c_{i j}\right|\right)_{n \times n}$. In addition, we denote by $I$ the identity matrix with compatible dimension, and denote $\|u\|_{2}^{2}=\int_{\Omega} u(t, x) d x$.

Lemma 1.1 Let $\varepsilon>0$ be any given scalar, and $\mathcal{M}$, $\mathfrak{E}$ and $\mathcal{K}$ be matrices with appropriate dimensions. If $\mathcal{K}^{T} \mathcal{K} \leq I$, then we have $\mathcal{M K} \mathfrak{E}+\mathfrak{E}^{T} \mathcal{K}^{T} \mathcal{M}^{T} \leq \varepsilon^{-1} \mathcal{M} \mathcal{M}^{T}+\varepsilon \mathfrak{E}^{T} \mathfrak{E}$.

Lemma $1.2\left(\left[2\right.\right.$, Lemma 6]) Let $P=\operatorname{diag}\left(p_{1}, p_{2}, \ldots, p_{n}\right)$ be a positive definite matrix, and $v$ be a solution of system (1.6) with boundary condition (1.1a). Then we have

$$
\begin{aligned}
\int_{\Omega} v^{T} P\left(\nabla \cdot\left(\mathcal{D}(t, x, v) \circ \nabla_{p} v\right)\right) d x & =-\sum_{k=1}^{m} \sum_{j=1}^{n} \int_{\Omega} p_{j} \mathcal{D}_{j k}(t, x, v)\left|\nabla v_{j}\right|^{p-2}\left(\frac{\partial v_{j}}{\partial x_{k}}\right)^{2} d x \\
& =\int_{\Omega}\left(\nabla \cdot\left(\mathcal{D}(t, x, v) \circ \nabla_{p} v\right)\right)^{T} P v d x .
\end{aligned}
$$

\section{Main result}

Theorem 2.1 Assume $p>1$. PDEs (1.6) is global stochastic exponential robust stability in the mean square if there exist a positive scalar $\beta>0$ and positive definite diagonal matrices $P_{i}(i \in S), L_{1}, L_{2}$ and $Q_{1}, Q_{2}$ such that for each $i \in S, j=1,2, \ldots, r$, the following $L M I$ conditions hold:

$$
\left(\begin{array}{cccccccc}
\frac{1}{r} a_{i 1} & 0 & 0 & \frac{1}{r} a_{i 4} & \frac{1}{r} a_{i 5} & \frac{1}{r} a_{i 6} & P_{i} \bar{A}\left|E_{i j}\right| & 0 \\
* & \frac{1}{r} a_{i 2} & 0 & 0 & \frac{1}{r}\left(G_{1}+G_{2}\right) L_{2} & 0 & 0 & 0 \\
* & * & \frac{1}{r} a_{i 3} & 0 & 0 & \frac{1}{r}\left(G_{1}+G_{2}\right) L_{2} & 0 & 0 \\
* & * & * & -\frac{2}{r} L_{1} & 0 & 0 & 0 & \left|H_{i j}^{T}\right| \\
* & * & * & * & -\frac{2}{r} L_{2} & 0 & 0 & c_{0}\left|M_{i j}^{T}\right| \\
* & * & * & * & * & -\frac{2}{r} L_{2} & 0 & \left(1-c_{0}\right)\left|M_{i j}^{T}\right| \\
* & * & * & * & * & * & -I & 0 \\
* & * & * & * & * & * & * & -I
\end{array}\right)<0
$$

where $a_{i 1}=-2 P_{i} \underline{A} \mathbb{B}-2 F_{1} L_{1} F_{2}+\beta P_{i}+\sum_{l \in S} \pi_{i l} P_{l}+P_{i} \Gamma_{1 i}+Q_{1}+Q_{2} ; a_{i 2}=P_{i} \Gamma_{2 i}-(1-$ $\left.a_{0}\right) e^{-\tau_{1 i} \beta} Q_{1}-2 G_{1} L_{2} G_{2} ; a_{i 3}=P_{i} \Gamma_{3 i}-\left(1-a_{0}\right) e^{-\tau_{2 i} \beta} Q_{2}-2 G_{1} L_{2} G_{2} ; a_{i 4}=\sum_{j=1}^{r} P_{i} \bar{A}\left|C_{i j}\right|+\left(F_{1}+\right.$ $\left.F_{2}\right) L_{1} ; a_{i 5}=c_{0} \sum_{j=1}^{r} P_{i} \bar{A}\left|D_{i j}\right| ; a_{i 6}=\left(1-c_{0}\right) \sum_{j=1}^{r} P_{i} \bar{A}\left|D_{i j}\right|$.

Proof Consider the Lyapunov-Krasovskii functional $V(t, i)=V_{1 i}+V_{2 i}, \forall i \in S$, where $V_{1 i}=e^{\beta t} \int_{\Omega} u^{T}(t, x) P_{i} u(t, x) d x$, and $V_{2 i}=e^{\beta t}\left[\int_{\Omega} \int_{-\tau_{1 i}(t)}^{0} e^{\beta \theta} u^{T}(t+\theta, x) Q_{1} u(t+\theta, x) d \theta d x+\right.$ $\left.\int_{\Omega} \int_{-\tau_{2 i}(t)}^{0} e^{\beta \theta} u^{T}(t+\theta, x) Q_{2} u(t+\theta, x) d \theta d x\right]$.

It follows immediately by Lemma 1.2 that $\int_{\Omega} u^{T} P_{i}\left(\nabla \cdot\left(\mathcal{D}(t, x, u) \circ \nabla_{p} u\right)\right) d x \leq 0$. 
Let $\mathscr{L}$ be the weak infinitesimal operator such that $\mathscr{L} V(t, u(t, x), i)=\mathscr{L} V_{1 i}+\mathscr{L} V_{2 i}$ for any given $i \in S$. Then we have

$$
\begin{aligned}
\mathscr{L} V_{1 i} \leq & -e^{\beta t}\left\{2 \int_{\Omega} u^{T} P_{i} \underline{A} \mathbb{B} u d x-2 \int_{\Omega} \sum_{j=1}^{r} h_{j}(\omega(t))\left[u^{T} P_{i} \bar{A} C_{i j}(t) f(u)\right.\right. \\
& +c_{0} u^{T} P_{i} \bar{A} D_{i j}(t) g\left(u\left(t-\tau_{1 i}(t), x\right)\right) \\
& \left.\left.+\left(1-c_{0}\right) u^{T} P_{i} \bar{A} D_{i j}(t) g\left(u\left(t-\tau_{2 i}(t), x\right)\right)\right] d x+\int_{\Omega} u^{T}\left(\sum_{l \in S} \pi_{i l} P_{l}\right) u d x\right\} \\
& +e^{\beta t} \int_{\Omega}\left[u^{T} P_{i} \Gamma_{1 i} u+u^{T}\left(t-\tau_{1 i}(t)\right) P_{i} \Gamma_{2 i} u\left(t-\tau_{1 i}(t)\right)\right. \\
& \left.+u^{T}\left(t-\tau_{2 i}(t)\right) P_{i} \Gamma_{3 i} u\left(t-\tau_{2 i}(t)\right)\right] d x+\beta e^{\beta t} \int_{\Omega} u^{T} P_{i} u d x, \\
\mathscr{L} V_{2 i} \leq & e^{\beta t}\left[\int_{\Omega} u^{T}\left(Q_{1}+Q_{2}\right) u d x\right. \\
& -\left(1-a_{0}\right) e^{-\tau_{1 i} \beta} \int_{\Omega} u^{T}\left(t-\tau_{1 i}(t), x\right) Q_{1} u\left(t-\tau_{1 i}(t), x\right) d x \\
& \left.-\left(1-a_{0}\right) e^{-\tau_{2 i} \beta} \int_{\Omega} u^{T}\left(t-\tau_{2 i}(t), x\right) Q_{2} u\left(t-\tau_{2 i}(t), x\right) d x\right] .
\end{aligned}
$$

From (A3), we have $2\left|f^{T}(u)\right| L_{1}|f(u)|-2\left|u^{T}\right|\left(F_{1}+F_{2}\right) L_{1}|f(u)|+2\left|u^{T}\right| F_{1} L_{1} F_{2}|u| \leq 0$, $2\left|g^{T}\left(u\left(t-\tau_{1 i}(t), x\right)\right)\right| L_{2}\left|g\left(u\left(t-\tau_{1 i}(t), x\right)\right)\right|+2\left|u^{T}\left(t-\tau_{1 i}(t), x\right)\right| G_{1} L_{2} G_{2}\left|u\left(t-\tau_{1 i}(t), x\right)\right| \leq$ $2\left|u^{T}\left(t-\tau_{1 i}(t), x\right)\right|\left(G_{1}+G_{2}\right) L_{2}\left|g\left(u\left(t-\tau_{1 i}(t), x\right)\right)\right|$, and $2\left|g^{T}\left(u\left(t-\tau_{2 i}(t), x\right)\right)\right| L_{2} \mid g\left(u\left(t-\tau_{2 i}(t)\right.\right.$, $x))|+2| u^{T}\left(t-\tau_{2 i}(t), x\right)\left|G_{1} L_{2} G_{2}\right| u\left(t-\tau_{2 i}(t), x\right)|\leq 2| u^{T}\left(t-\tau_{2 i}(t), x\right)\left|\left(G_{1}+G_{2}\right) L_{2}\right| g(u(t-$ $\left.\left.\tau_{2 i}(t), x\right)\right) \mid$.

Combining the above inequalities results in $\mathscr{L} V(t, i) \leq e^{\beta t} \int_{\Omega} \zeta^{T}(t, x) \mathfrak{A}_{i} \zeta(t, x) d x$, where $\zeta(t, x)=\left(\left|u^{T}(t, x)\right|,\left|u^{T}\left(t-\tau_{1 i}(t), x\right)\right|,\left|u^{T}\left(t-\tau_{2 i}(t), x\right)\right|,\left|f^{T}(u(t, x))\right|,\left|g^{T}\left(u\left(t-\tau_{1 i}(t), x\right)\right)\right|\right.$, $\left.\left|g^{T}\left(u\left(t-\tau_{2 i}(t), x\right)\right)\right|\right)^{T}$,

$$
\mathfrak{A}_{i}=\left(\begin{array}{cccccc}
a_{i 1} & 0 & 0 & \tilde{a}_{i 4} & \tilde{a}_{i 5} & \tilde{a}_{i 6} \\
& a_{i 2} & 0 & 0 & \left(G_{1}+G_{2}\right) L_{2} & 0 \\
& * & a_{i 3} & 0 & 0 & \left(G_{1}+G_{2}\right) L_{2} \\
* & * & -2 L_{1} & 0 & 0 \\
* & * & * & -2 L_{2} & 0 \\
* & * & * & * & -2 L_{2}
\end{array}\right),
$$

and $\tilde{a}_{i 4}=\sum_{j=1}^{r} h_{j}(\omega(t)) P_{i} \bar{A}\left|C_{i j}(t)\right|+\left(F_{1}+F_{2}\right) L_{1}, \tilde{a}_{i 5}=c_{0} \sum_{j=1}^{r} h_{j}(\omega(t)) P_{i} \bar{A}\left|D_{i j}(t)\right|, \tilde{a}_{i 6}=(1-$ $\left.c_{0}\right) \sum_{j=1}^{r} h_{j}(\omega(t)) P_{i} \bar{A}\left|D_{i j}(t)\right|$.

Further, we can apply the Schur complement [4] to (2.1), and derive $\mathfrak{A}_{i}<0$ by Lemma 1.1. Hence, $\mathscr{L} V(t, i) \leq 0$. Define $\mathcal{V}(t, i)=\int_{\Omega} u^{T}(t, x) P_{i} u(t, x) d x+\int_{\Omega} \int_{-\tau_{i}(t)}^{0} e^{\beta \theta} u^{T}(t+\theta, x) \times$ $Q u(t+\theta, x) d \theta d x$. From the Dynkin formula, we can derive that $e^{\beta t} \mathbb{E} \mathcal{V}(t)-\mathbb{E} \mathcal{V}(0)=$ $\mathbb{E} \int_{0}^{t} \mathscr{L}\left(e^{\beta s} \mathcal{V}(s)\right) d s \leq 0$. Now, for any $\phi(\theta, x) \in L_{\mathcal{F}_{0}}^{2}\left([-\tau, 0] \times \Omega ; R^{n}\right)$ and any system mode $i \in S$, the solution $u\left(t, x, \phi, i_{0}\right)$ of system (1.6) with the initial value $\phi$ satisfies $\min _{i \in S}\left\{\underline{\alpha}_{i}\right\} e^{\beta t} \mathbb{E}\left(\left\|u\left(t, x, \phi, i_{0}\right)\right\|_{2}^{2}\right) \leq\left(\max _{i \in S}\left\{\bar{\alpha}_{i}\right\}+\lambda_{\max } Q\right) \sup _{-\tau \leq \theta \leq 0} \mathbb{E}\left(\|\phi(\theta)\|_{2}^{2}\right), \forall t \geq 0$, or $\mathbb{E}\left(\left\|u\left(t, x ; \phi, i_{0}\right)\right\|_{2}^{2}\right) \leq \gamma e^{-\beta t} \sup _{-\tau \leq \theta \leq 0} \mathbb{E}\left(\|\phi(\theta, x)\|_{2}^{2}\right), \forall t \geq 0$, where positive scalars $\underline{\alpha}_{i}, \bar{\alpha}_{i}$ satisfy $\underline{\alpha}_{i} I \leq P_{i}$ and $\bar{\alpha} I \geq P_{i}$ for any mode $i \in S$, scalars $\gamma=\frac{1}{\min _{i \in S}\left\{\underline{\alpha}_{i}\right\}}\left(\max _{i \in S}\left\{\bar{\alpha}_{i}\right\}+\lambda_{\max } Q\right)>0$, 
$\beta>0$. Therefore, PDEs (1.6) is global stochastic exponential robust stability in the mean square.

Remark 2.1 As pointed out in [1], diffusion effect exists really in the neural networks when electrons are moving in asymmetric electromagnetic fields [5]. Strictly speaking, reaction-diffusion terms should be considered in any neural networks model [6-8]. Usually, the diffusion behaviors were simulated by linear Laplace diffusion items [9-16]. But not all diffusion behaviors can be simply considered as the linear reaction-diffusion. Indeed, there are various works related to the nonlinear reaction-diffusion [17-21], and even the nonlinear $p$-Laplace diffusion $[17,20]$. So, in this paper, the stability of $p$-Laplace PDEs was investigated.

Example 2.1 Consider PDEs (1.6) with the following parameters: $\underline{A}=I_{2}=\bar{A}, B=1.5 I_{2}$, $F_{1}=G_{1}=0, F_{2}=G_{2}=0.1 I_{2}, \beta=0.01, r=2, S=\{1,2\}, \pi_{11}=-0.8, \pi_{12}=0.8, \pi_{21}=0.5$, $\pi_{22}=-0.5, c_{0}=0.9, a_{0}=0.1$. Let $i=1,2 ; j=1,2 ; k=1,2,3$, and $C_{i j}=D_{i j}=0.1 I_{2}, \tau_{2 i}=380$, $\tau_{1 i}=10, \Gamma_{k i} \equiv 0.01 I_{2}, E_{i j}=H_{i j}=M_{i j}=0.01 I_{2}$. By using Matlab LMI toolbox, we solve LMI condition (2.1) and obtain $t_{\min }=-0.0149<0$, which implies feasible (see [2, Remark 29(3)] for details). Further, one can extract data as follows:

$$
\begin{array}{ll}
P_{1}=\left(\begin{array}{cc}
15.8743 & 0 \\
0 & 15.8743
\end{array}\right), & P_{2}=\left(\begin{array}{cc}
15.6796 & 0 \\
0 & 15.6796
\end{array}\right), \\
Q_{1}=\left(\begin{array}{cc}
4.9980 & 0 \\
0 & 4.9980
\end{array}\right), & Q_{2}=\left(\begin{array}{cc}
25.9273 & 0 \\
0 & 25.9273
\end{array}\right), \\
L_{1}=\left(\begin{array}{cc}
4.8023 & 0 \\
0 & 4.8023
\end{array}\right), & L_{2}=\left(\begin{array}{cc}
0.5294 & 0 \\
0 & 0.5294
\end{array}\right) .
\end{array}
$$

Then Theorem 2.1 derives that PDEs (1.6) is global stochastic exponential robust stability in the mean square with a large allowable variation range of time delay $[0,380]$.

Remark 2.2 To the best of our knowledge, it is the first attempt to investigate the robust stability of T-S fuzzy Markovian jumping Itô-type stochastic dynamic equations with $p$ Laplace and probabilistic time-varying delays (see [1, 2, 20, 22-25]). Example 2.1 shows the effectiveness of the proposed methods due to the large allowable variation range of time delay.

Remark 2.3 As pointed out in [26], almost all the above related literature did not point out the role that the nonlinear $p$-Laplace items play, except [1] and [20]. In fact, when $p=2$, 2-Laplace is the linear Laplace, and there are many papers (see, e.g., [10-13]) in which the Laplace diffusion item plays its role in their stability criteria, for the linear Laplace PDEs can be considered in the special Hilbert space $H^{1}(\Omega)$ that can be orthogonally decomposed into the direct sum of infinitely many eigenfunction spaces. However, the nonlinear $p$-Laplace $(p>1, p \neq 2)$ brings great difficulties for the nonlinear $p$-Laplace PDEs should be considered in the frame of the Sobolev space $W^{1, p}(\Omega)$ that is only a reflexive Banach space. Indeed, owing to the great difficulties, the authors only provide in [1] and [20] the stability criterion in which the nonlinear $p$-Laplace items play roles in the case of $1<p<2$ and $p>1$ under the Dirichlet boundary condition. So, a further profound study is very 
interesting, which may call for some new mathematical methods, and even new mathematical theories. Under the Neumann boundary condition, the problem of the role of the nonlinear $p$-Laplace $(p>1)$ item in the stability criteria for fuzzy stochastic $p$-Laplace PDEs with probabilistic delays still remains open and challenging.

\section{Competing interests}

The authors declare that they have no competing interests.

\section{Authors' contributions}

$\mathrm{XW}$, the first author of this manuscript, carried out the main part of this manuscript. RR is the corresponding author of this manuscript. All authors typed, read and approved the final manuscript.

\section{Acknowledgements}

This work is supported by the Scientific Research Fund of Science Technology Department of Sichuan Province (2011 JYZ010, 2012JYZ010), and by the Scientific Research Fund of Sichuan Provincial Education Department (11ZA172, 14ZA0274, 12ZB349).

\section{Received: 27 August 2014 Accepted: 6 November 2014 Published: 26 Nov 2014}

\section{References}

1. Rao, RF, Zhong, SM, Wang, XR: Stochastic stability criteria with LMI conditions for Markovian jumping impulsive BAM neural networks with mode-dependent time-varying delays and nonlinear reaction-diffusion. Commun. Nonlinear Sci. Numer. Simul. 19(1), 258-273 (2014)

2. Rao, RF, Wang, XR, Zhong, SM, Pu, ZL: LMI approach to exponential stability and almost sure exponential stability for stochastic fuzzy Markovian-jumping Cohen-Grossberg neural networks with nonlinear p-Laplace diffusion. J. Appl. Math. 2013, Article ID 396903 (2013)

3. Xu, D, Li, B, Long, S, Teng, L: Moment estimate and existence for solutions of stochastic functional differential equations. Nonlinear Anal., Theory Methods Appl. 108, 128-143 (2014)

4. Boyd, SP, Ghaoui, LF, Feron, F, Balakrishnan, V: Linear Matrix Inequalities in Systems and Control Theory. SIAM, Philadelphia (1994)

5. Liao, X, Yang, S, Chen, S, Fu, Y: Stability of general neural networks with reaction-diffusion. Sci. China, Ser. F 44(5), 389-395 (2001)

6. Kao, Y, Wang, C, Karimi, HR, Bi, R: Global stability of coupled Markovian switching reaction-diffusion systems on networks. Nonlinear Anal. Hybrid Syst. 13, 61-73 (2014)

7. Song, Q, Cao, J: Dynamics of bidirectional associative memory networks with distributed delays and reaction-diffusion terms. Nonlinear Anal., Real World Appl. 8(1), 345-361 (2007)

8. Song, Q, Cao, J: Global exponential robust stability of Cohen-Grossberg neural network with time-varying delays and reaction-diffusion terms. J. Franklin Inst. 343(7), 705-719 (2006)

9. Wang, L, Zhang, Z, Wang, Y: Stochastic exponential stability of the delayed reaction-diffusion recurrent neural networks with Markovian jumping parameters. Phys. Lett. A 372(18), 3201-3209 (2008)

10. Pan, J, Zhong, S: Dynamical behaviors of impulsive reaction-diffusion Cohen-Grossberg neural network with delays. Neurocomputing 73, 1344-1351 (2010)

11. Pan, J, Liu, X, Zhong, S: Stability criteria for impulsive reaction-diffusion Cohen-Grossberg neural networks with time-varying delays. Math. Comput. Model. 51, 1037-1050 (2010)

12. Pan, J, Zhong, S: Dynamic analysis of stochastic reaction-diffusion Cohen-Grossberg neural networks with delays. Adv. Differ. Equ. 2009, Article ID 410823 (2009)

13. Pan, J, Zhong, S: Novel criteria on global robust exponential stability to a class of reaction-diffusion neural networks with delays. Discrete Dyn. Nat. Soc. 2009, Article ID 291594 (2009)

14. Wang, K, Teng, Z, Jiang, H: Global exponential synchronization in delayed reaction-diffusion cellular neural networks with the Dirichlet boundary conditions. Math. Comput. Model. 52(1-2), 12-24 (2010)

15. Zhang, X, Wu, S, Li, K: Delay-dependent exponential stability for impulsive Cohen-Grossberg neural networks with time-varying delays and reaction-diffusion terms. Commun. Nonlinear Sci. Numer. Simul. 16(3), 1524-1532 (2011)

16. Gan, Q, Xu, R, Yang, P: Exponential synchronization of stochastic fuzzy cellular neural networks with time delay in the leakage term and reaction-diffusion. Commun. Nonlinear Sci. Numer. Simul. 17(4), 1862-1870 (2012)

17. Chen, J, Guo, J: Image restoration based on adaptive P-Laplace diffusion. In: International Congress on Image and Signal Processing (CISP2010), pp. 143-146 (2010)

18. Baranwal, VK, Pandey, RK, Tripathi, MP, Singh, OP: An analytic algorithm for time fractional nonlinear reaction-diffusion equation based on a new iterative method. Commun. Nonlinear Sci. Numer. Simul. 17(10), 3906-3921 (2012)

19. Meral, G, Tezer-Sezgin, M: The comparison between the DRBEM and DQM solution of nonlinear reaction-diffusion equation. Commun. Nonlinear Sci. Numer. Simul. 16(10), 3990-4005 (2011)

20. Rao, RF, Pu, ZL, Zhong, SM, Huang, JL: On the role of diffusion behaviors in stability criterion for $p$-Laplace dynamical equations with infinite delay and partial fuzzy parameters under Dirichlet boundary value. J. Appl. Math. 2013, Article ID 940845 (2013)

21. Cherniha, R, Davydovych, $\mathrm{V}$ : Conditional symmetries and exact solutions of nonlinear reaction-diffusion systems with non-constant diffusivities. Commun. Nonlinear Sci. Numer. Simul. 17(8), 3177-3188 (2012)

22. Pan, QF, Zhang, ZF, Huang, JC: Stability of the stochastic reaction-diffusion neural network with time-varying delays and p-Laplacian. J. Appl. Math. 2012, Article ID 405939 (2012). doi:10.1155/2012/405939 
23. Rao, R, Zhong, S, Wang, X: Delay-dependent exponential stability for Markovian jumping stochastic Cohen-Grossberg neural networks with $p$-Laplace diffusion and partially known transition rates via a differential inequality. Adv. Differ. Equ. 2013, Article ID 183 (2013)

24. Rao, R: Delay-dependent exponential stability for nonlinear reaction-diffusion uncertain Cohen-Grossberg neural networks with partially known transition rates via Hardy-Poincaré inequality. Chin. Ann. Math., Ser. B 35, 575-598 (2014)

25. Rao, R, Pu, Z: Stability analysis for impulsive stochastic fuzzy p-Laplace dynamic equations under Neumann or Dirichlet boundary condition. Bound. Value Probl. 2013, Article ID 133 (2013)

26. Pu, ZL, Rao, RF: Exponential robust stability of TS fuzzy stochastic $p$-Laplace PDEs under zero-boundary condition. Bound. Value Probl. 2013, Article ID 264 (2013)

10.1186/1029-242X-2014-469

Cite this article as: Wang and Rao: Robust stability of probabilistic delays fuzzy stochastic $p$-Laplace dynamic equations. Journal of Inequalities and Applications 2014, 2014:469

Submit your manuscript to a SpringerOpen ${ }^{\circ}$ journal and benefit from:

- Convenient online submission

- Rigorous peer review

- Immediate publication on acceptance

- Open access: articles freely available online

- High visibility within the field

- Retaining the copyright to your article 